\title{
Optimal Graph Search Segmentation Using Arc-Weighted Graph for Simultaneous Surface Detection of Bladder and Prostate ${ }^{\star}$
}

\author{
Qi Song ${ }^{1}$, Xiaodong $\mathrm{Wu}^{1,2}$, Yunlong $\mathrm{Liu}^{1}$, Mark Smith ${ }^{2}$, John Buatti ${ }^{2}$, \\ and Milan Sonka ${ }^{1,2,3}$ \\ 1 Department of Electrical \& Computer Engineering \\ 2 Department of Radiation Oncology \\ 3 Department of Ophthalmology \& Visual Sciences, \\ University of Iowa, Iowa City, IA 52242, USA \\ \{qi-song, xiaodong-wu, milan-sonka\}@uiowa.edu
}

\begin{abstract}
We present a novel method for globally optimal surface segmentation of multiple mutually interacting objects, incorporating both edge and shape knowledge in a 3-D graph-theoretic approach. Hard surface interacting constraints are enforced in the interacting regions, preserving the geometric relationship of those partially interacting surfaces. The soft smoothness a priori shape compliance is introduced into the energy functional to provide shape guidance. The globally optimal surfaces can be simultaneously achieved by solving a maximum flow problem based on an arc-weighted graph representation. Representing the segmentation problem in an arc-weighted graph, one can incorporate a wider spectrum of constraints into the formulation, thus increasing segmentation accuracy and robustness in volumetric image data. To the best of our knowledge, our method is the first attempt to introduce the arcweighted graph representation into the graph-searching approach for simultaneous segmentation of multiple partially interacting objects, which admits a globally optimal solution in a low-order polynomial time. Our new approach was applied to the simultaneous surface detection of bladder and prostate. The result was quite encouraging in spite of the low saliency of the bladder and prostate in CT images.
\end{abstract}

\section{Introduction}

In the United States, prostate cancer is one of the most common cancers in men, accounting for about $25 \%$ of all newly diagnosed cases [1. Precise target delineation is critical for a successful 3-D radiotherapy treatment planning for prostate cancer treatment. Automatic segmentation techniques are urgently needed due to large amounts of 3-D image data that require increased time for manually contouring.

\footnotetext{
* This work was supported in part by NSF grants CCF-0830402 and CCF-0844765, and NIH grants R01-EB004640 and K25-CA123112. We would like to thank Dr. Honghai Zhang' help with pre-segmentation of prostate CT image data.
} 
The segmentation of pelvic structure is of particularly difficulty. It involves soft tissues that present a large variability in shape and size. Those soft tissues also have similar intensity and have seriously mutual influence in position and shape. Many attempts have been tried in this area, such as registration approach (e.g. [2]), implicit and explicit models (e.g. 345]), and bayesian formulation (e.g. 6]). None of these methods can produce a globally optimal solution with respect to a task-specific objective function. Freedman et al. [7] developed an interactive approach based on graph cut method 8]. Their method incorporating soft shape prior allows for a global optimum. However, it focuses on only single object (bladder) segmentation and is at least non-trivial to incorporate mutually interacting surface constraints to simultaneously segment multiple object surfaces. We are thus motivated to propose a novel method incorporating both edge and shape information for globally optimal segmentation of bladder and prostate.

Our work is a non-trivial extension of the framework proposed by $\mathrm{Wu}$ et al. [9] and Li et al. 10]. Instead of only employing node weights in the graph to represent the desired segmentation properties, we propose in this paper an arcweighted graph representation, which utilizes the weights of both graph nodes and arcs to incorporate a wider spectrum of constraints, e.g., the soft smoothness a priori shape compliance presented in this paper and other prior shape knowledge. Hard surface interacting constraints are enforced in interacting regions to preserve geometric relationships between partially interacting boundary surfaces of prostate and bladder. Soft smoothness shape compliance is further employed to incorporate shape information. Two mutually interacting optimal surfaces are then computed by solving a maximum flow problem.

Yin et al. 11] have developed a framework based on the graph-searching approach for knee-joint segmentation of bones and cartilages. Their work yet was still limited to using the node-weighted graph representation. Li et al. [12] adopted the arc-weighted graph representation to incorporate the elliptic shape model priors and smoothness penalty for simultaneous delineation multiple surfaces of a single object. Most recently, graph-cut based segmentation methods have attracted a lot of attention. One of the most influential work is Boykov and Funka-Lea's interactive segmentation algorithm for $d$-D images based on minimum $s$ - $t$ cuts [8], which is topologically flexible and shares some elegance with the level set methods. However, incorporating a priori shapes and simultaneous detection of mutually interacting surfaces into the Boykov framework and any other previous graph-based methods is at least non-trivial. Also, unlike the graph cut method, our proposed method requires less/no human interaction.

\section{Optimal Segmentation of Multiple Mutually Interacting Objects Incorporating Both Edge and Shape Knowledge}

To present our method in a comprehensible manner, in this section we consider the task of detecting a terrain-like surface representing the boundary of a 
3-D object in a volumetric image. Note that the simpler principles used for this illustration are directly applicable to arbitrarily-irregularly meshed surfaces. We introduce a soft smoothness a priori shape compliance term into the energy function using an arc-weighted graph model to substantially extended the graphsearching framework 910]. The extension makes it possible to incorporate a wider spectrum of constraints.

\subsection{Problem Formulation}

Consider a volumetric image $\mathcal{I}(X, Y, Z)$ of size $X \times Y \times Z$. For each $(x, y)$ pair, the voxel subset $\{\mathcal{I}(x, y, z) \mid 0 \leq z<Z\}$ forms a column parallel to the z-axis, denoted by $\operatorname{Col}(x, y)$. Each column has a set of neighborhoods for a certain neighbor setting, e.g., four-neighbor relationship 10. The surface of particularly interest in $\mathcal{I}$, denoted $S(x, y)$, is the terrain-like surface which intersects with exactly one voxel of each column of voxels parallel to the z-axis. A surface is considered feasible if it satisfies certain smoothness constraints. Specifically, if $\mathcal{I}\left(x_{1}, y_{1}, z_{1}\right)$ and $\mathcal{I}\left(x_{2}, y_{2}, z_{2}\right)$ are two voxels on the surface from neighboring columns in the $x$-direction, then $\left|z_{1}-z_{2}\right| \leq \Delta_{x}$, where $\Delta_{x}$ is a specified smoothness parameter. Similar constraints exist for neighboring columns in the y-direction.

Let $\Gamma=[0 . . X-1] \times[0 . . Y-1]$ denote the grid domain of image $\mathcal{I}$. We enforce a surface interacting constraints for each pair of the sought surfaces $S_{i}$ and $S_{j}$ in mutually interacting region $R_{i j} \subseteq \Gamma$, on which both $S_{i}$ and $S_{j}$ are interacting each other. For any $(x, y) \in R_{i j}$, if $\mathcal{I}(x, y, z) \in S_{i}$ and $\mathcal{I}\left(x, y, z^{\prime}\right) \in S_{j}$, then we have $\delta_{i j}^{l} \leq z^{\prime}-z \leq \delta_{i j}^{u}$, where $\delta_{i j}^{l} \geq 0$ and $\delta_{i j}^{u} \geq 0$ are two specified surface interacting constraints. An edge-based cost $c_{i}(x, y, z)$ is assigned to each voxel $\mathcal{I}(x, y, z)$ for each target surface $S_{i}$, which is inversely related to the likelihood that the desired surface contains the voxel. Yet utilizing only image edge information may not be sufficient. To make full use of priori information and incorporate a wider spectrum of constraints, we introduce into the objective function a soft smoothness a priori shape compliance energy term $\mathcal{E}_{\text {smooth }}(S)$ for surface $S$, with $\mathcal{E}_{\text {smooth }}(S)=\int_{\Gamma} \phi(\nabla S)$, where $\phi$ is a smoothness penalty function. Assume that $\mathcal{N}$ is a given neighborhood system on $\Gamma$. For any $p(x, y) \in \Gamma$, let $S(p)$ denote the z-coordinate of the voxel $\mathcal{I}(x, y, z)$ on $S$. Then, the discrete form of a priori shape compliance smoothness energy $\mathcal{E}_{\text {smooth }}(S)$ can be expressed as $\Sigma_{(p, q) \in \mathcal{N}} f_{p, q}(|S(p)-S(q)|)$, where $f_{p, q}$ is a non-decreasing function associated with two neighboring columns of $p$ and $q$ that penalizes the shape changes of $S$ on $p$ and $q$. Then our enhanced optimal surface detection (EOSD) problem seeks an optimal set $S$ of $\lambda$ surfaces in $\mathcal{I}$ such that (1) each individual surface satisfies hard smoothness constraints; (2) each pair of surfaces satisfies surface interacting constraints; and (3) the $\operatorname{cost} \alpha(S)$ induced by $S$, with the form

$$
\alpha(S)=\sum_{i=1}^{\lambda} \sum_{I_{i}(x, y, z) \in S_{i}} c_{i}(x, y, z)+\sum_{i=1}^{\lambda} \sum_{(p, q) \in \mathcal{N}} f_{p, q}^{(i)}\left(\left|S_{i}(p)-S_{i}(q)\right|\right)
$$

is minimized. In this paper, we focus on the linear smoothness penalty functions, i.e., $f_{p, q}(h)=a \cdot h+b(a$ and $b$ are a constant, $h=0,1,2, \ldots)$ between two neighboring columns $\operatorname{Col}(p)$ and $\operatorname{Col}(q)$. 


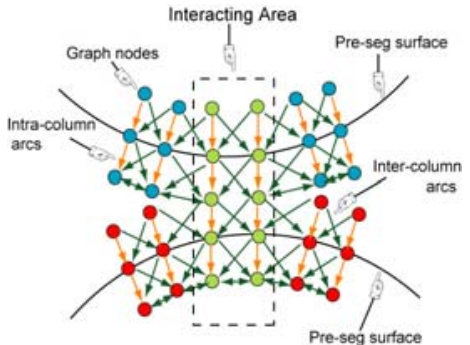

(a)

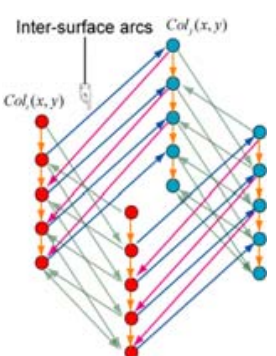

(b)

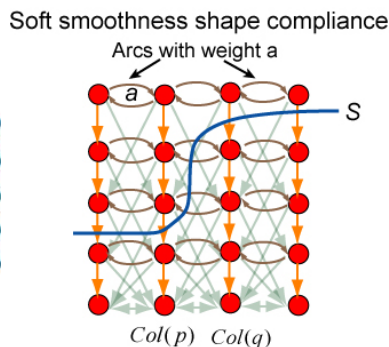

(c)

Fig. 1. Graph construction for mutually interacting objects. An example 2-D slice from a 3-D image is presented. (a) Intra-column arcs (yellow arrows) and inter-column arcs(green arrows). The hard smoothness parameter is set 1. (b) Inter-surface arcs (blue and red arrows) on the interacting region. For visualization, the slice on the interacting region is duplicated and visualized in two separated slices. The distance between two surfaces ranges from $\delta_{i j}^{l}=1$ to $\delta_{i j}^{u}=2$. (c) Incorporating a linear smoothness penalty. The feasible surface $S$ cuts the arcs with a total weight of $2 a$ on the neighboring columns $\operatorname{Col}(p)$ and $\operatorname{Col}(q)$, which measures the smoothness shape compliance term of $\alpha(S)$ for the two neighboring columns. Green arrow shows the hard smoothness constraints (inter-column arcs) with parameter 2 .

\subsection{The Arc-Weighted Graph Representation for Incorporating $A$ Priori Shape Knowledge}

In the original graph-searching model 910, only the node weights in a graph was used to represent the desired segmentation properties, e.g., edge-based image costs. To incorporate a prior shape knowledge, we utilize the weights of both graph nodes and arcs (directed graph edges) to represent the desired segmentation properties. The basic idea is to reduce the EOSD problem to the minimum $s$-excess problem [9. A directed graph $G$ containing $\lambda$ node-disjoint subgraphs $\left\{G_{i}=\left(V_{i}, E_{i}\right): i=1,2, \ldots, \lambda\right\}$ is defined, in which every node $V_{i}(x, y, z) \in V_{i}$ represents exactly one voxel $\mathcal{I}(x, y, z)$. Both intra-column arcs and inter-column arcs are added to ensure the monotonicity of the target surfaces and the hard smoothness constraints (Fig[1(a)), as described in 10. To enforce the surface interacting constraints between any two sought surfaces $S_{i}$ and $S_{j}$ on the given mutually interacting region $R_{i j}$, the inter-surface arcs with $+\infty$ weights are added (Fig[1(b)). Suppose for the two sought surfaces $S_{i}$ and $S_{j}$, the prior knowledge puts $S_{i}$ below $S_{j}$ and the distance between $S_{i}$ and $S_{j}$ ranges from $\delta_{i j}^{l}$ to $\delta_{i j}^{u}$. A directed arc is put from each node $V_{j}(x, y, z)$ with $(x, y) \in R_{i j}$ and $z<Z-\delta_{i j}^{l}$ to node $V_{i}\left(x, y, z+\delta_{i j}^{l}\right)$ in $G_{i}$. This ensures that if voxel $\mathcal{I}(x, y, z)$ lies on surface $S_{j}$, then the voxel $\mathcal{I}\left(x, y, z^{\prime}\right)$ on $S_{i}$ must be no "lower" than voxel $\mathcal{I}\left(x, y, z+\delta_{i j}^{l}\right)$ (i.e., $\left.z^{\prime} \geq z+\delta_{i j}^{l}\right)$. On the other hand, each node $V_{i}(x, y, z)$ with $(x, y) \in R_{i j}$ and $z \geq \delta_{i j}^{l}$ has an arc to $V_{j}\left(x, y, z^{\prime}\right)$ with $z^{\prime}=\max \left\{0, z-\delta_{i j}^{u}\right\}$, making sure that if $\mathcal{I}(x, y, z)$ is on $S_{i}$, then the voxel of $\operatorname{Col}(x, y)$ on $S_{j}$ must be no "lower" than voxel $\mathcal{I}\left(x, y, z^{\prime}\right)$. To incorporate the linear smoothness penalty, for any two 
neighboring columns $\operatorname{Col}(p)$ and $\operatorname{Col}(q)$ with $p=(x, y)$ and $q=\left(x^{\prime}, y^{\prime}\right)$, node $V_{i}(x, y, z)$ has one arc to node $V_{i}\left(x^{\prime}, y^{\prime}, z\right)$ as well as one arc from $V_{i}\left(x^{\prime}, y^{\prime}, z\right)$ to $V_{i}(x, y, z)$, each with an arc-weight of $a$ for all $z=1, \ldots, Z-1$. If a feasible surface $S_{i}$ intersects columns $\operatorname{Col}(p)$ and $\operatorname{Col}(q)$ at $S_{i}(p)$ and $S_{i}(q)$, respectively, a total weight of $a \cdot\left(\left|S_{i}(p)-S_{i}(q)\right|\right)$ contributes to the corresponding cut in $G$, which is equivalent to the soft smoothness penalty $f_{p, q}^{(i)}\left(\left|S_{i}(p)-S_{i}(q)\right|\right)$ for the minimization problem while considering that $b$ is a constant for any feasible surface $S_{i}(\operatorname{Fig} \mathbb{1}(\mathrm{c}))$. Thus, the linear smoothness penalty is incorporated.

The weight of each node in the graph is set as described in 10. With this constructed graph $G$, an optimal cut $\mathcal{C}^{*}=\left(A^{*}, \bar{A}^{*}\right)\left(A^{*} \cup \bar{A}^{*}=V\right)$ in $G$ minimizing the total weight of nodes in $A^{*}$ plus the total arc weight of $\mathcal{C}^{*}$ defines an optimal set $\mathcal{S}^{*}$ of $\lambda$ surfaces in $\mathcal{I}$ minimizing the objective function $\alpha\left(\mathcal{S}^{*}\right)$. Note that $\mathcal{C}^{*}$ can be computed efficiently using the maximum flow algorithm in a low-order polynomial time [9]. The optimal $\lambda$ surfaces can then be recovered by computing the upper envelope of the optimal cut $\mathcal{C}^{*}$.

\section{Simultaneous Surface Detection of Bladder and Prostate}

In this section, we apply our optimal graph searching method developed in Section 2 for simultaneous segmentation of bladder and prostate in 3-D CT images. Our approach mainly consists of two stages: (a) Pre-segmentation of the objects of interest. An approximation of target surfaces can be obtained, which gives useful information about the topological structures of the target objects. (b) Accurate delineation using graph optimization based on the pre-segmented surface mesh. The use of surface mesh allows our method to readily incorporate shape priors and the known topology.

\subsection{Pre-segmentation of Bladder and Prostate}

A 3-D geodesic active contour method [13] was conducted for pre-segmentation of the bladder. Three user-defined points were required as an initial input. The prostate shows a much better coherency in shape than bladder. Hence we computed the mean shape of the prostate from the training set of eight 3-D manual segmentation. Then an approximate bounding box of interest for prostate is interactively defined and the obtained mean shape was roughly fitted into the never-before seen CT images using rigid transformations as the pre-segmentation result. Note that the pre-segmentation results only serve to provide a basic topological structure information, thus we do not require accurate segmentation at this stage. The statement was proved in the following experiments. Overlapping between pre-segmented prostate and bladder is also allowed, which can be resolved in the graph optimization step.

\subsection{Graph Construction and Optimization}

From pre-segmentation results, two triangulated meshes were generated using isosurfacing algorithm (e.g., marching cubes) to specify neighboring relations 
among voxels on sought surfaces. As described in Section 2.2, to utilize our arc-weighted graph representation for mutually interacting surface detection, a one-to-one correspondence between two surface meshes needed to be computed on "interacting regions", which were defined according to the distance between two pre-segmented surfaces. Note that prostate and bladder are always almost attached to each other or with a very small distance away. Thus we simply projected the pre-segmented prostate surface mesh on the interacting region to the mesh of the pre-segmented bladder boundary surface. Then we use the projected mesh patch to replace the original bladder surface mesh on the interacting region. Thus, a one-to-one mesh correspondence on interacting region was established since two new meshes on that area have exactly the same topological structure.

For each vertex of the two triangulated meshes, a vector of voxels (columns) was created. The number of nodes on each column depends on the required resolution. For columns outside the interacting region, the normal at each mesh vertex was used as the column (sampling) direction. For each mesh vertex on the interacting region, a column of voxels that captures both prostate and bladder boundaries, was computed along the average normal of the pre-segmented prostate mesh for enforcing the surface interacting constrains between the bladder and prostate boundaries.

A weighted directed graph $G$ was then constructed as in Section 2.2. To incorporate the shape priori knowledge, we employed a linear soft smoothness penalty $f_{p, q}(h)=a \cdot h+b(h=0,1,2, \ldots)$ between two neighboring columns $\operatorname{Col}(p)$ and $\operatorname{Col}(q)$, where $a$ and $b$ are constant parameters. In our experiments, we set $a=5$ and $b=0$ according to the experimental results on the training set.

Cost function design plays an important role for successful surface detection. In our segmentation, the gradient-based cost function was employed for edge-based costs. The negative magnitude of the gradient of the image $\mathcal{I}$ was computed at each voxel as $c_{e d g e}=|\nabla \mathcal{I}|$.

Observe that intensities of bladder and prostate are generally higher than surrounding tissues. A Sobel kernel was used to favor a bright-to-dark transition.

\section{Experiments and Results}

21 3-D CT images from different patients with prostate cancer were employed for validation. No contrast agent was filled. 8 datasets were randomly selected as the training set to build a shape model of prostate. Segmentation experiments were carried out on the remaining 13 datasets. The resolution of image ranges from $0.98 \times 0.98 \times 3.00 \mathrm{~mm}^{3}$ to $1.60 \times 1.60 \times 3.00 \mathrm{~mm}^{3}$.

The computed results were compared with the expert tracing outlines. We employed the methods similiar to those used in [3] for the quantitative measures, which include the following: (1) The probability of detection $v_{d}$, calculated as the fraction of the expert-defined target object that was contained by our computed result; (2) The probability of false alarm $v_{f a}$, calculated as the fraction of our computed target object that lies outside the expert-defined result; and (3) The unsigned surface distance error $s_{d}$, computed as the distance between the expert-defined surface and our computed surface, which was expressed 
Table 1. Overall quantitative results for the bladder-prostate segmentation

\begin{tabular}{cccclll} 
& \multicolumn{3}{c}{ With soft smoothness } & \multicolumn{3}{c}{ Without soft smoothness } \\
\hline & $v_{d}$ & $v_{f a}$ & $s_{d}(\mathrm{~mm})$ & $v_{d}$ & $v_{f a}$ & $s_{d}(\mathrm{~mm})$ \\
\hline Bladder & 0.958 & 0.115 & $1.04 \pm 1.00$ & 0.958 & 0.120 & $1.09 \pm 1.02$ \\
Prostate & 0.852 & 0.136 & $1.38 \pm 1.08$ & 0.800 & 0.122 & $1.48 \pm 1.17$ \\
\hline
\end{tabular}

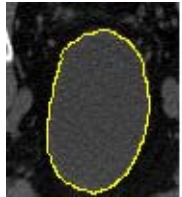

(a)

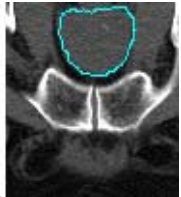

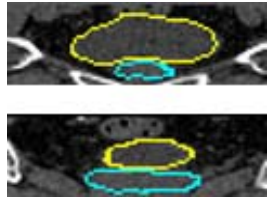

(b)

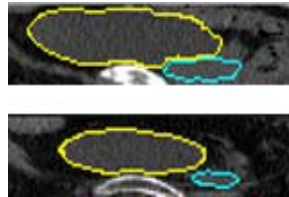

(c)

Fig. 2. The bladder (yellow) and prostate (blue) segmentation results. (a) Transverse view. (b) Coronal view. (c) Sagittal view.
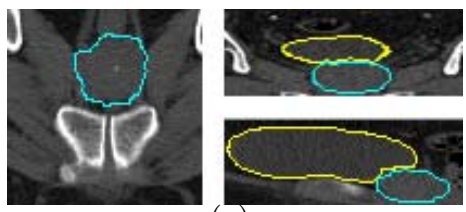

(a)
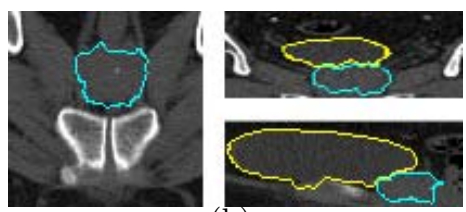

(b)

Fig. 3. Comparison of simultaneous bladder-prostate segmentation. (a) Linear smoothness penalty used. (b) No soft smoothness penalty. Identical hard smoothness constraints and cost functions were used.

as mean \pm standard deviation. To test the effectiveness of using soft smoothness a priori shape compliance energy, experiments were conducted for all image datasets with and without using the soft-smoothness term.

The result is shown in Table. 1. Our approach generally produced a highquality segmentation (i.e., $v_{d}$ near $0.9, v_{f a}$ near 0.1 ), which is similar to the inter-expert variability as reported in 514]. By using the soft smoothness term, segmentation results for prostate were obviously improved in 9 out of 13 datasets. The improvement for bladder was relatively small.

Illustrative results of our experiments are shown in Fig. 2(a)-(c) for three views. From all views, our surface interacting constraints work quite well with no overlapping of prostate and bladder on the interacting region. Fig. 3 shows the comparison of simultaneous bladder-prostate segmentation segmentation results with and without soft smoothness term, exhibiting expected improvement.

A nature question is that how sensitive the final segmentation is to presegmentation result. To answer this question, two different experiments were conducted. First, pre-segmentation results of bladder and prostate were perturbed by 2 voxels separately in each of the in-plane directions, resulting in 12 pre-segmented results for each dataset. Second, pre-segmentation results were 
scaled up by a factor of 1.1 and scaled down by 0.9 for the bladder and the prostate separately, resulting in 4 more pre-segmentions for each dataset. The experiments were done on one randomly chosen dataset. For all 16 perturbed and scaled pre-segmentations, the final segmentations remain successful, and the mean unsigned surface distance error was $1.10 \mathrm{~mm}$ for bladder and $1.13 \mathrm{~mm}$ for prostate, which are comparable to the original results: $1.09 \mathrm{~mm}$ for bladder and $1.07 \mathrm{~mm}$ for prostate.

\section{Discussion and Conclusion}

In this paper, we introduce a novel graph-searching method using an arc-weighted graph representation for simultaneous segmentation of bladder and prostate. Our method provides a general framework for simultaneous segmentation of multiple partially interacting surfaces belonging to multiple objects in an optimal fashion with respect to a task-specific objective function. The arc-weighted graph representation allows an easy incorporation of a wider spectrum of constraints while utilizing weighted combinations of edge- and shape-based costs. 13 3-D CT datasets were employed for validation. The result is quite impressive considering the difficulty of the segmentation for pelvic structures.

\section{References}

1. Jemal, A., Siegel, R., Ward, E., Hao, Y., Murray, T., Thun, M.J.: Cancer statistics. Lancet 353, 267-272 (2008)

2. Malsch, U., Thieke, C., Bendl, R.: Fast elastic registration for adaptive radiotherapy. In: Larsen, R., Nielsen, M., Sporring, J. (eds.) MICCAI 2006. LNCS, vol. 4191, pp. 612-619. Springer, Heidelberg (2006)

3. Freedman, D., Radke, R.J., Zhang, T., Jeong, Y., Lovelock, D.M., Chen, G.T.Y.: Model-based segmentation of medical imagery by matching distributions. IEEE Trans. Medical Imaging 24(3) (2005)

4. Dam, E., Fletcher, P.T., Pizer, S.M., Tracton, G., Rosenman, J.: Prostate shape modeling based on principal geodesic analysis bootstrapping. In: Barillot, C., Haynor, D.R., Hellier, P. (eds.) MICCAI 2004. LNCS, vol. 3217, pp. 1008-1016. Springer, Heidelberg (2004)

5. Costa, M.J., Delingette, H., Novellas, S., Ayache, N.: Automatic segmentation of bladder and prostate using coupled 3D deformable models. In: Ayache, N., Ourselin, S., Maeder, A. (eds.) MICCAI 2007, Part I. LNCS, vol. 4791, pp. 252-260. Springer, Heidelberg (2007)

6. Rousson, M., Khamene, A., Diallo, M.H., Celi, J.C., Sauer, F.: Constrained surface evolutions for prostate and bladder segmentation in CT images. In: Liu, Y., Jiang, T.-Z., Zhang, C. (eds.) CVBIA 2005. LNCS, vol. 3765, pp. 251-260. Springer, Heidelberg (2005)

7. Freedman, D., Zhang, T.: Interactive graph cut based segmentation with shape priors. In: CVPR 2005, vol. 1, pp. 755-762 (2005)

8. Boykov, Y., Funka-Lea, G.: Graph cuts and efficient N-D image segmentation. Int. Journal of Computer Vision 70(2), 109-131 (2006) 
9. Wu, X., Chen, D.Z.: Optimal net surface problems with applications. In: Widmayer, P., Triguero, F., Morales, R., Hennessy, M., Eidenbenz, S., Conejo, R. (eds.) ICALP 2002. LNCS, vol. 2380, p. 1029. Springer, Heidelberg (2002)

10. Li, K., Wu, X., Chen, D.Z., Sonka, M.: Optimal surface segmentation in volumetric images - a graph-theoretic approach. IEEE Trans. Pattern Anal. Machine Intell. 28(1), 119-134 (2006)

11. Yin, Y., Zhang, X., Sonka, M.: Optimal multi-object multi-surface graph search segmentation: full-joint cartilage delineation in 3D. In: MIUA 2008, pp. 104-108 (2008)

12. Li, K., Jolly, M.P.: Simultaneous detection of multiple elastic surfaces with application to tumor segmentation in CT images. In: SPIE 2008, vol. 6914 (2008)

13. Caselles, V., Kimmel, R., Sapiro, G.: Geodesic active contours. International journal on computer vision 22, 61-97 (1997)

14. Fiorino, C., Reni, M., Bolognesi, A., Cattaneo, G.M., Calandrino, R.: Intra- and inter- observer variability in contouring prostate and seminal vesicles: Implications for conformal treatment planning. Radiotherapy Oncol. 47(3), 285-292 (1998) 